SHEPHERD ET AL

\title{
NMR Assignments
}

\section{Table of Resonances}

Table 1. ${ }^{1} \mathrm{H}$ NMR resonance assignments and chemical shifts ( $\left.\delta \mathrm{ppm}\right)$ for $\mathbf{1}$ in $90 \% \mathrm{H}_{2} \mathrm{O}: 10 \% \mathrm{D}_{2} \mathrm{O}$ (310 K pH 4.2).

\begin{tabular}{|c|c|c|c|c|}
\hline Residue & $\mathrm{NH}$ & $\mathrm{H} \alpha$ & $\mathrm{H} \beta$ & Other \\
\hline Ac-Phe1 & 8.23 & 4.86 & $3.09,2.95$ & Ar-H 7.2-7.4 \\
\hline Pro2 & & 4.49 & $2.25,2.07$ & $\mathrm{H} \gamma 2.00, \mathrm{H \delta} 3.87,3.47$ \\
\hline Lys3 & 8.20 & 4.17 & $1.97,1.80$ & $\begin{array}{l}\mathrm{H} \gamma 1.26, \mathrm{H} \delta 1.68,1.52, \mathrm{H \varepsilon} 3.78 \\
2.65, \mathrm{NH} \zeta 8.12\end{array}$ \\
\hline Asp4 & 8.60 & 4.44 & 2.86 & \\
\hline Glu5 & 7.75 & 4.14 & $2.47,2.40$ & Hg 2.08 \\
\hline Phe6 & 8.44 & 4.18 & 3.07 & Ar-H 7.2-7.4 \\
\hline Asp7 & 9.04 & 4.47 & 2.76 & \\
\hline Lys8 & 7.61 & 4.15 & $1.99,1.91$ & $\begin{array}{l}\mathrm{H} \gamma 1.21, \mathrm{H} \delta 1.58,1.38, \mathrm{H \varepsilon} 3.65 \\
2.58, \mathrm{NH} \zeta 7.97\end{array}$ \\
\hline Ser9 & 7.83 & 4.15 & 2.56 & \\
\hline Ile10 & 7.32 & 3.83 & 1.82 & $\begin{array}{l}\mathrm{H} \gamma\left(\mathrm{CH}_{2}\right) 1.27,1.01, \mathrm{H} \gamma\left(\mathrm{CH}_{3}\right) \\
0.78, \mathrm{H} \delta, 0.66\end{array}$ \\
\hline Arg11 & 7.57 & 4.14 & $1.88,1.85$ & $\begin{array}{l}\mathrm{H} \gamma 1.27,1.01, \mathrm{H} \delta 3.21, \mathrm{NH} \varepsilon \\
7.27\end{array}$ \\
\hline Asp12 & 8.08 & 4.73 & $2.93,2.68$ & \\
\hline Val13-NH & 7.52 & 4.07 & 2.17 & $\mathrm{H} \gamma 0.99$ \\
\hline
\end{tabular}


2. Comparison of Ha chemical shifts

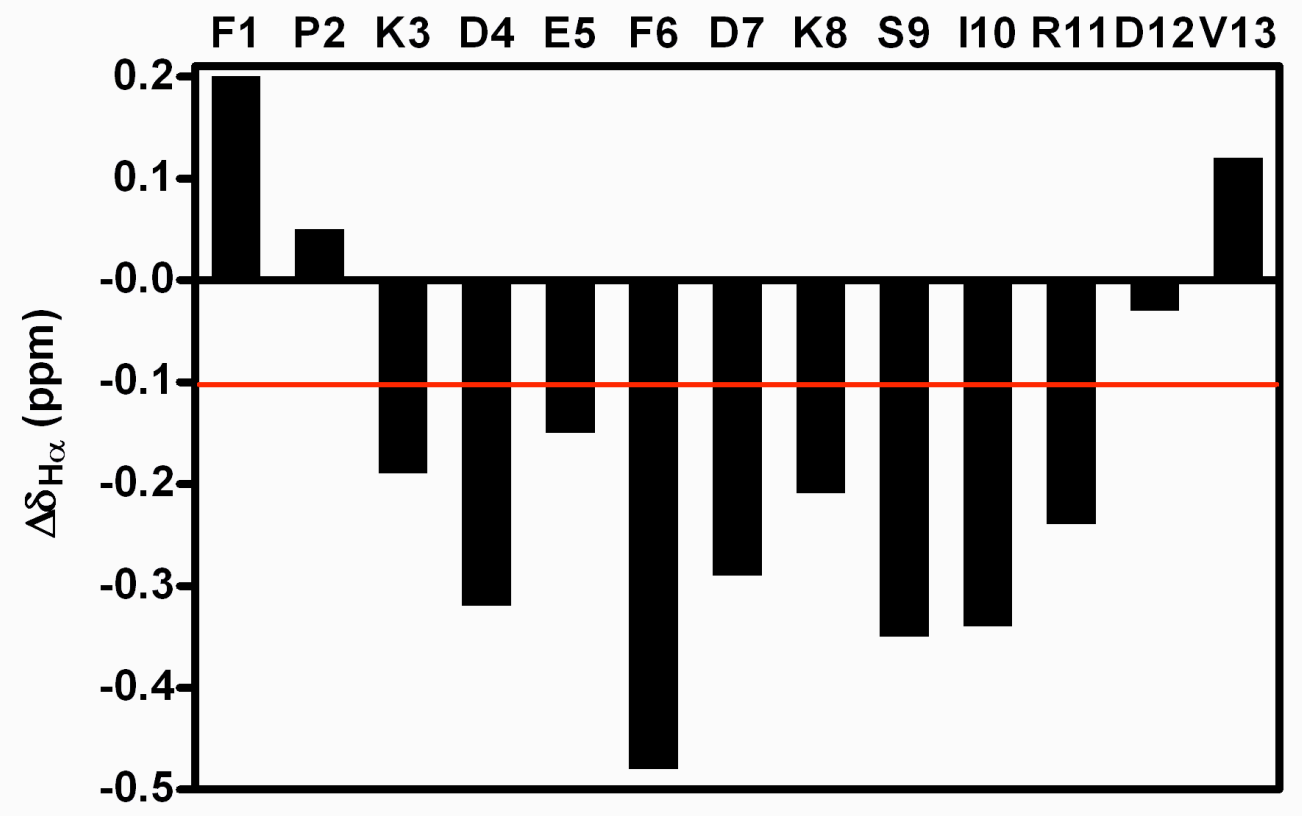

Figure 1. Differences between observed chemical shift and random coil values ${ }^{6}\left(\Delta \delta=\delta-\delta_{\text {random }}\right)$ for 1 in $\mathrm{H}_{2} \mathrm{O} / \mathrm{D}_{2} \mathrm{O}(9: 1)$. Negative stretches $>0.1 \mathrm{ppm}$ (red line) are observed for helical residues. 


\section{Spectra and Assignments}

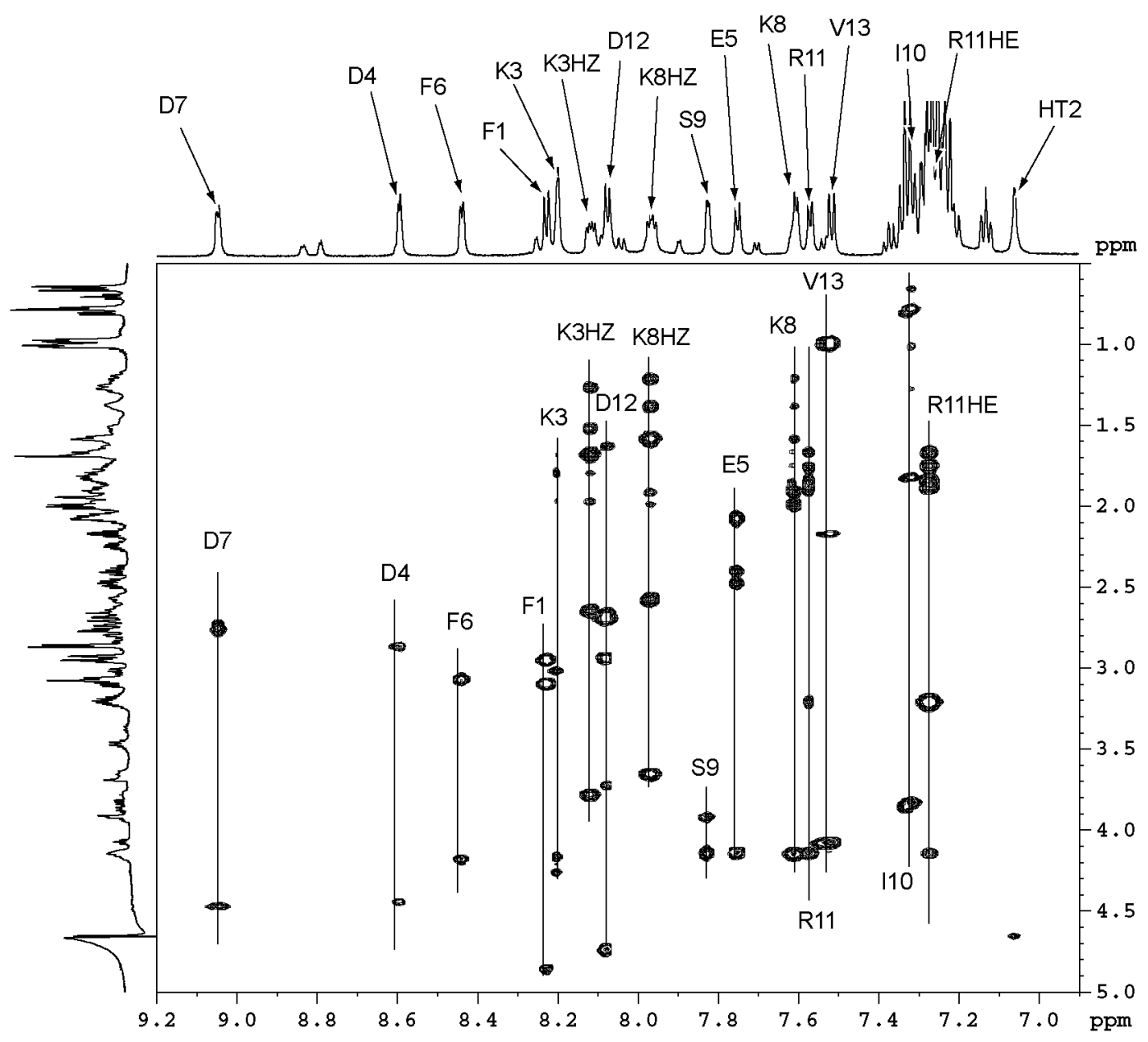

Figure 2. Fingerprint region from the $600 \mathrm{MHz}$ TOCSY spectrum for $\mathbf{1}$ in $\mathrm{H}_{2} \mathrm{O}: \mathrm{D}_{2} \mathrm{O}(9: 1)$ at $310 \mathrm{~K}$. Spin systems are indicated by solid lines and are labelled according to standard one letter amino acid codes and by their sequence position in $\mathbf{1}$. 


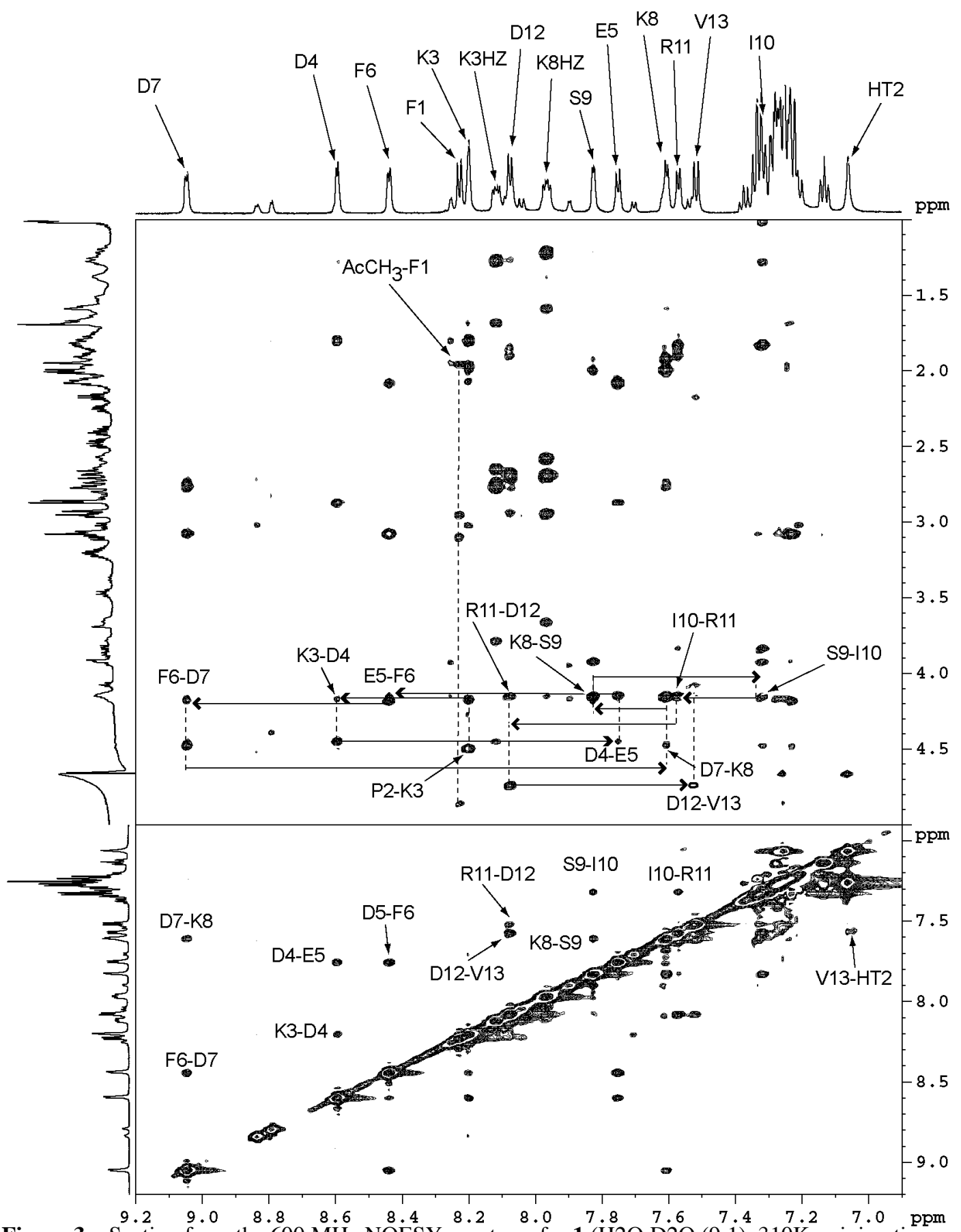

Figure 3. . Section from the $600 \mathrm{MHz}$ NOESY spectrum for 1 (H2O:D2O $(9: 1), 310 \mathrm{~K}$, mixing time $300 \mathrm{~ms}$ ). Sequential connectivity for $\mathbf{1}$. Intra-residue $\mathrm{NH}-\mathrm{CH}$ cross-peaks are labelled according to standard one letter amino acid codes and by their residue numbers in $\mathbf{1}$

\section{Structure Calculation Information}

\section{Phi Angle restraints}




\section{SHEPHERD ET AL}

Table 2. Amide ${ }^{3} \mathrm{~J}_{\mathrm{HN}-\mathrm{H} \alpha}$ coupling constants for $\mathbf{1}$ in $\mathrm{H}_{2} \mathrm{O} / \mathrm{D}_{2} \mathrm{O}(9: 1)$ at $310 \mathrm{~K}$, and Phi angle restraints used in structure calculation.

\begin{tabular}{ccc}
\hline Residue & ${ }^{3} J_{\mathrm{NHCH \alpha}}(\mathrm{Hz})$ & $\phi$-restraints \\
\hline Phe1 & 7.0 & NA \\
Lys3 & 2.0 & $-65.0 \pm 30^{\circ}$ \\
Asp4 & 3.1 & $-65.0 \pm 30^{\circ}$ \\
Glu5 & 5.9 & $-65.0 \pm 30^{\circ}$ \\
Phe6 & 4.8 & $-65.0 \pm 30^{\circ}$ \\
Asp7 & 5.3 & $-65.0 \pm 30^{\circ}$ \\
Lys8 & 3.0 & $-65.0 \pm 30^{\circ}$ \\
Ser9 & 5.7 & $-65.0 \pm 30^{\circ}$ \\
Ile10 & 6.6 & NA \\
Arg11 & 5.1 & $-65.0 \pm 30^{\circ}$ \\
Asp12 & 6.4 & NA \\
Val13 & 6.6 & NA \\
\hline
\end{tabular}

\section{Hydrogen bond restraints}

Table 3. Changes in the chemical shift of amide protons in 1 over $278-318 \mathrm{~K}_{\text {in }} \mathrm{H}_{2} \mathrm{O} / \mathrm{D}_{2} \mathrm{O}(9: 1)$. Hydrogen bonds are inferred for temperature coefficients less than $4 \mathrm{ppb} / \mathrm{K}$.

$\begin{array}{ccc}\text { Residue } & \Delta \delta / \mathrm{T}(\mathrm{ppb} / \mathrm{K}) & \text { H-bond } \\ & \mathrm{H}_{2} \mathrm{O} / \mathrm{D}_{2} \mathrm{O}(9: 1) & (\Delta \delta / \mathrm{T}<4 \mathrm{ppb} / \mathrm{K})\end{array}$

\begin{tabular}{ccc}
\hline Phe1 & 9.2 & $\mathrm{~N}$ \\
\hline Lys3 & 3.3 & $\mathrm{Y}$ \\
\hline Asp4 & 3.6 & $\mathrm{Y}$ \\
\hline Glu5 & 6.2 & $\mathrm{~N}$ \\
\hline Phe6 & 5.2 & $\mathrm{~N}$ \\
\hline Asp7 & 8.1 & $\mathrm{~N}$ \\
\hline Lys8 & 4.1 & $\mathrm{~N}$ \\
\hline Ser9 & 1.0 & $\mathrm{Y}$
\end{tabular}


SHEPHERD ET AL

\begin{tabular}{ccc}
\hline Ile10 & $*$ & $\mathrm{~N}$ \\
\hline Arg11 & 2.5 & $\mathrm{Y}$ \\
\hline Asp12 & 2.8 & $\mathrm{Y}$ \\
\hline Val13 & 0.5 & $\mathrm{Y}$ \\
\hline HT1 & $*$ & $\mathrm{~N}$ \\
\hline HT2 & $*$ & $\mathrm{~N}$ \\
\hline
\end{tabular}

* could not be determined due to spectra overlap with aromatic signals.

\section{Distance restraints used in final XPLOR calculation}

Table 4. NOE derived distance restraints used for calculating the solution structure of compound $\mathbf{1}$ in $90 \% \mathrm{H}_{2} \mathrm{O}: 10 \% \mathrm{D}_{2} \mathrm{O}$.

\begin{tabular}{|c|c|c|}
\hline Atom A & Atom B & Upper distance restraint (A) and comment. \\
\hline $\mathrm{Ac}-\mathrm{CH}_{3}$ & Phe2-NH & 4.2; Strong $+1.5 \AA$ correction \\
\hline Phe2-H $\alpha$ & Pro3-H_\# & 4.5; Medium $+1.0 \AA$ correction \\
\hline Phe2-H $\alpha$ & Asp5-H $\beta \#$ & $6.0 ;$ Weak + $1.0 \AA$ correction \\
\hline Pro3-H $\alpha$ & Lys4-NH & 3.5; Medium \\
\hline Pro3-H $\alpha$ & Asp5-NH & 5.0; Weak \\
\hline Pro3-H $\alpha$ & Phe7-NH & 5.0; Weak \\
\hline Pro3-H $\beta \#$ & Lys4-NH & 4.5; Medium $+1.0 \AA$ correction \\
\hline Lys4-NH & Asp5-NH & 2.7; Strong \\
\hline Lys4-Ha & Asp5-NH & 5.0; Weak \\
\hline Lys4-Ha & Glu6-NH & 5.0; Weak \\
\hline Lys4-Ha & Phe7-NH & 6.0 ; Very weak \\
\hline Lys4-H $\beta \#$ & Asp5-NH & 4.5; Medium + $1.0 \AA$ correction \\
\hline Lys4-H_\# & Asp5-NH & 4.5; Medium + $1.0 \AA$ correction \\
\hline Lys4-H $\gamma \#$ & Asp5-NH & 6.0 ; Weak + 1.0 Å correction \\
\hline Asp5-NH & Glu6-NH & 2.7; Strong \\
\hline Asp5-NH & Phe7-NH & 5.0; Weak \\
\hline Asp5-H $\alpha$ & Glu6-NH & 3.5; Medium \\
\hline Asp5-H $\alpha$ & Phe7-NH & 6.0 ; Very weak \\
\hline
\end{tabular}




\begin{tabular}{|c|c|c|}
\hline Asp5-H $\alpha$ & Asp8-NH & 6.0 ; Very weak \\
\hline Asp5-Hß\# & Glu6-NH & 3.7; Strong + 1.0 Å correction \\
\hline Glu6-NH & Phe7-NH & 2.7; Strong \\
\hline Glu6-NH & Phe7- H $\beta \#$ & 7.0; Very Weak + $1.0 \AA$ A correction \\
\hline Glu6-H $\alpha$ & Lys9-NH & 6.0 ; Very weak \\
\hline Glu6-H $\beta \#$ & Phe7-NH & 3.7; Strong + 1.0 Å correction \\
\hline Phe7-NH & Asp8-NH & 2.7; Strong \\
\hline Phe7-NH & Lys9- Hß\# & $6.0 ;$ Weak $+1.0 \AA$ correction \\
\hline Phe7- H $\alpha$ & Asp8-NH & 3.5; Medium \\
\hline Phe7- H $\alpha$ & Lys9-NH & 6.0 ; Very weak \\
\hline Phe7- H $\alpha$ & Ser10-NH & 5.0; Weak \\
\hline Phe7- H $\alpha$ & Ser11-NH & 6.0 ; Very weak \\
\hline Phe7- H $\beta \#$ & Asp8-NH & 3.7; Strong $+1.0 \AA$ correction \\
\hline Asp8-NH & Lys9-NH & 2.7; Strong \\
\hline Asp8-NH & Ser10-NH & 5.0; Weak \\
\hline Asp8-H $\alpha$ & Lys9-NH & 3.5; Medium \\
\hline Asp8-H $\alpha$ & Ile11-NH & 3.5; Medium \\
\hline Asp8-H $\alpha$ & Ile11- H $\beta$ & 3.5; Medium \\
\hline Asp8-H $\alpha$ & Arg12-NH & 5.0; Weak \\
\hline Asp8-H $\beta \#$ & Lys9-NH & 4.5; Medium $+1.0 \AA$ A correction \\
\hline Lys9-NH & Ser10-NH & 2.7; Strong \\
\hline Lys9-NH & Ser10- H $\beta \#$ & 5.0; Weak \\
\hline Lys9-H $\beta \#$ & Ser10-NH & 3.7; Strong + $1.0 \AA$ A correction \\
\hline Lys9-H_\# & Ser10-NH & $6.0 ;$ Weak $+1.0 \AA$ A correction \\
\hline Lys9-H $\gamma \#$ & Ser10-NH & 6.0; Weak + 1.0 Å correction \\
\hline Ser10-NH & Ile11-NH & 2.7; Strong \\
\hline Ser10-NH & Arg12-NH & 5.0; Weak \\
\hline Ser10-H $\alpha$ & Asp13-NH & 5.0; Weak \\
\hline Ser10- $H \beta \#$ & Asp13-NH & 6.0 ; Very weak \\
\hline Ile11-NH & Arg12-NH & 2.7; Strong \\
\hline Ile11-NH & Asp13-NH & 5.0; Weak \\
\hline Ile11-H $\alpha$ & Arg12-NH & 5.0; Weak \\
\hline
\end{tabular}




\section{SHEPHERD ET AL}

$\begin{array}{lcc}\text { Ile11-H } \alpha & \text { Asp13-NH } & 6.0 ; \text { Very weak } \\ \text { Ile11-H } \alpha & \text { Val14-NH } & 6.0 ; \text { Very weak } \\ \text { Arg12-NH } & \text { Asp13-NH } & 2.7 ; \text { Strong } \\ \text { Arg12-NH } & \text { Val14-NH } & 5.0 ; \text { Weak } \\ \text { Asp13-NH } & \text { Val14-NH } & 2.7 ; \text { Strong } \\ \text { Asp13-H } \alpha & \text { Val14-NH } & 5.0 ; \text { Weak } \\ \text { Asp13-H } \beta \# & \text { Val14-NH } & 6.0 ; \text { Weak }+1.0 \AA \text { correction }\end{array}$

